\title{
Spontaneous Spiral Dissection of the Left Anterior Descending Coronary Artery in a Reformed Smoker
}

\author{
Debasish Das ${ }^{1}$, Debasis Acharya ${ }^{2}$, Jogendra Singh ${ }^{3}$, Subhas Pramanik ${ }^{4}$ \\ From ${ }^{1}$ Associate Professor, ${ }^{2}$ Assistant Professor, ${ }^{3}$ Senior Resident, ${ }^{4}$ Senior Cath Lab Technician, Department of Cardiology, All India Institute of \\ Medical Sciences, Bhubaneswar, Odisha, India
}

\begin{abstract}
Spontaneous dissection of the coronary artery is a well-known entity in young females with myocardial infarction. We report a rare case of spontaneous spiral dissection of the left anterior descending coronary artery (LAD) in a 62-year-old male with history of smoking for 15 years and who has quit smoking 5 years back. Just like an old age saying "footprints are left behind," the deleterious conundrum of smoking continues even after years together resulting in a spontaneous dissection in the background of damaged arterial elastic media.
\end{abstract}

Keywords: Coronary, Dissection, Spiral, Smoking

$\mathrm{S}$ pontaneous dissection of the coronary artery is a wellknown entity in young females presenting with acute myocardial infarction. Spontaneous dissection of the coronary artery accounts for $0.1 \%-0.4 \%$ of all the cases of the acute coronary syndrome (ACS) [1]. It accounts for $25 \%$ of all ACS cases in young females $<50$ years of age [2]. In Mayo Clinic Registry, no definite cause was identified in $45 \%$ of cases with spontaneous coronary artery dissection (SCAD) [3].

The most common predisposing factors are postpartum state, fibromuscular dysplasia, connective tissue disorder, and hormonal therapy in females. Extreme physical exertion, emotional stress, coughing, retching, vomiting, and use of sympathomimetic drugs such as cocaine and amphetamine are known as potential stressors behind SCAD [4]. The natural history of smokers resembles a normal person after 5 years of abstinence from smoking. We report a rare case of spontaneous spiral dissection of the left anterior descending coronary artery in a reformed smoker which has not been reported so far in the literature.

\section{CASE REPORT}

62-year-old male presented with complaints of acute anterior wall ST elevated myocardial infarction. The person was a well-known smoker for the past 15 years and was smoking 10 cigarettes per day (one packet), that is, 15 pack-years but he had quit smoking

\section{Access this article online}

Received - 17 March 2021

Initial Review - 02 April 2021

Accepted - 12 April 2021

DOI: 10.32677/IJCR.2021.v07.i04.010
5 years back. He was non-diabetic, non-hypertensive, and non-dyslipidemic.

Clinical examination revealed that he had a blood pressure of $100 / 60 \mathrm{mmHg}$ in the right arm supine position with a heart rate of 120 beats per minute and was regular in nature. He was mildly tachypneic with a respiration rate of $32 /$ minute and cardiovascular system examination revealed the presence of the third $\left(\mathrm{LVS}_{3)}\right.$ and fourth heart sounds $\left(\mathrm{LVS}_{4}\right)$ with fine bibasal crackles over the lung fields and systemic oxygen saturation $\left(\mathrm{SpO}_{2}\right)$ of $92 \%$.

Echocardiography revealed regional wall motion abnormality in the left anterior descending artery territory with mild left ventricular systolic dysfunction and was subjected to transradial coronary angiogram (CAG). CAG revealed long segment spiral dissection from osteoproximal to distal LAD without any evidence of atherosclerotic plaque or calcification (Fig. 1a-c).

Such a long segment spiral dissection of the coronary artery in reformed smokers is rare to see in clinical practice. In view of long segment spiral dissection, which may later develop a completely occlusive thrombus resulting in recurrent myocardial infarction with risk of sudden cardiac death, we gently crossed the lesion with Balanced Middleweight (BMW) 0.014" coronary guidewire and directly stented the lesion with $2.5 \times 32 \mathrm{~mm}$ drug-eluting stent (DES) at 14 atm pressure with the good angiographic result and distal TIMI III flow (Fig. 2). Although, small SCAD can be managed with antiplatelets, anticoagulants, and anti-ischemic medications, we stented the dissected segment in view of the long segment of involvement with the risk of future thrombosis.

The patient was discharged with dual antiplatelet therapy including ticagrelor, high-dose statin, and high-dose

Correspondence to: Dr. Debasish Das, Associate Professor and HOD, Department of Cardiology, All India Institute of Medical Sciences (AIIMS), Bhubaneswar, Odisha - 751 019, India. E-mail: dasdebasish54@gmail.com

(C) 2021 Creative Commons Attribution-NonCommercial 4.0 International License (CC BY-NC-ND 4.0). 


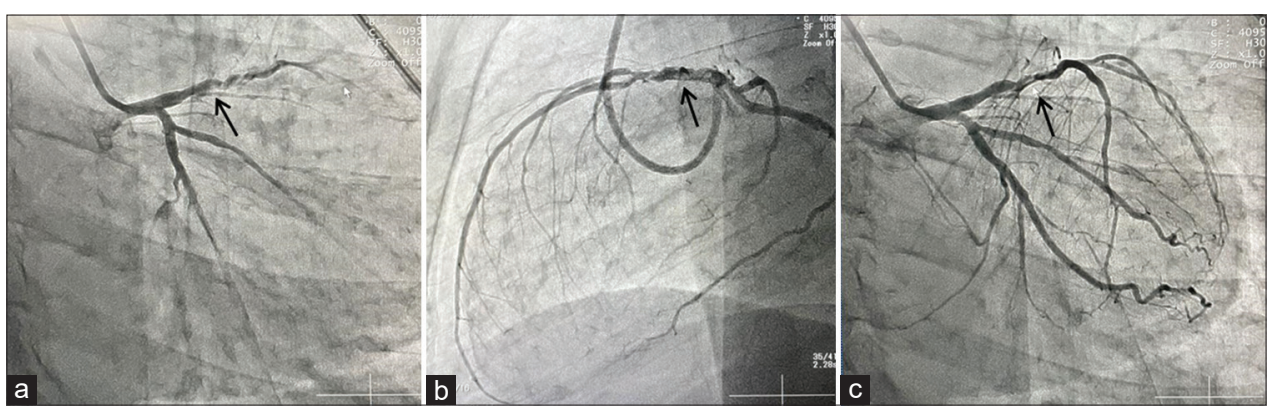

Figure 1: (a) RAO cranial $30^{\circ}$ showing spiral dissection beginning in proximal segment; (b) lateral view showing spiral dissection in mid and distal LAD; (c) guide shoot revealing long nature of spiral dissection of LAD

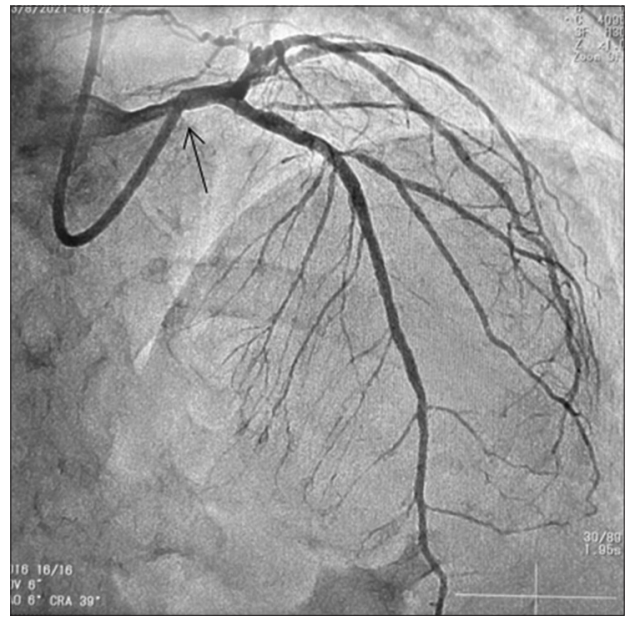

Figure 2: Post-stenting revealing good angiographic result

beta-blocker to decrease the arterial shear stress and prevent future spontaneous dissection of the coronary artery. A careful history of the patient revealed that there was no potential stressor behind the development of this spontaneous dissection in this reformed smoker; damaged elastic media of the coronary artery due to long smoking may be the plausible explanation behind it.

\section{DISCUSSION}

About $50 \%$ of SCAD present with ST elevated myocardial infarction and the rest present with non-ST elevated myocardial infarction with an elevated risk of life-threatening ventricular arrhythmia and sudden cardiac death [5].

Spontaneous coronary artery dissections are classified into three types as described by Saw et al [6]: Type 1: Double lumen appearance with a longitudinal filling defect, Type 2: Long tubular lesion $>30 \mathrm{~mm}$ with intramural hematoma, and Type 3: Multiple tubular lesions with intramural hematoma. Types 2 and 3 coronary dissections are diagnosed in intravascular ultrasound and optical coherence tomography (OCT). Our case was unique in presentation with a long segment spiral dissection that does not fit with any of the categories mentioned above.

Eleid et al. demonstrated that SCAD is associated with more arterial tortuosity ( $78 \%$ vs. $1.7 \%$ in controls) and severe arterial tortuosity is a marker of recurrent dissection [7]. SCAD is more common in LAD and multivessel involvement is seen in up to $20-25 \%$ of cases. Fibromuscular dysplasia is notorious to produce recurrent spontaneous coronary artery dissections out of all the predisposing factors mentioned [8]. SCAD can be also extremely catastrophic to cause sudden cardiac death (SCD) [9]. SCAD can involve multivessels including the left main coronary artery also [10].

Percutaneous coronary artery revascularization has been the mainstay of management and revealed successful outcome [11]. Although the role of fibrinolytic therapy in the acute phase of SCAD has been reported [12], therapeutic paradoxes in SCAD remain as not to use fibrinolytic therapy and GP IIb IIIa inhibitors in those cases as those increase the size of intramural hematoma and further extend the dissection.

Sudden complete occlusion of the left main or left anterior descending coronary artery may lead to ischemic ventricular fibrillation and also sudden cardiac arrest [13]. Female SCAD patients have a poorer prognosis, particularly postpartum patients were found to have larger infarcts, lower mean LV ejection fraction ( $34 \%$ vs. $49 \% ; P<0.01)$, and proximal artery dissections [14].

Technical challenges during percutaneous transluminal coronary intervention include avoiding deep engagement of the guide catheter due to the fragile nature of the coronary artery, gentle contrast injection to prevent dissection propagation, wiring the true lumen as an inadvertent entry of the wire into the false lumen extends the dissection with side branch occlusion [15]; gentle wiring with the workhorse wire-like BMW, Sion blue, or run through is always recommended.

We report this unique case of spontaneous spiral coronary artery dissection in a reformed smoker which we carefully revascularized with DES with the good angiographic result and distal TIMI III flow. Although the long-term prognosis is good in those cases, recurrent SCAD is higher with an average rate of $5 \%$ per year. To minimize the shear stress in coronary arteries, a high-dose beta-blocker is a must in long-term follow-up in those cohorts of patients. After 1 month of follow-up, our patient was doing well without any exertional shortness of breath or effort or rest angina with resolved ST-T changes in the ECG and normalized left ventricular ejection fraction. We advised the patient monthly follow-up for the first 6 months and then 3 monthly follow-up for the next year with strict adherence to antiplatelets, highdose statin, and beta-blocker without any miss to prevent future recurrence. 


\section{CONCLUSION}

We report a rare case of spontaneous long segment spiral dissection of LAD in a reformed smoker which has not been described in the literature so far. Although predisposing factors and potential stressors play a role behind the development of SCAD, it may be the consequence of damaged elastic media of the coronary artery due to the long history of smoking; "Smoking has left its footprint in the coronary artery resulting in a late spontaneous dissection."

\section{REFERENCES}

1. Mortensen KH, Thuesen L, Kristensen IB, Christiansen EH. Spontaneous coronary artery dissection: A western Denmark Heart Registry Study. Catheter Cardiovasc Interv 2009;74:710-7.

2. Saw J, Aymong E, Mancini GB, Sedlak T, Starovoytov A, Ricci D. Nonatherosclerotic coronary artery disease in young women. Can J Cardiol 2014;30:814-9.

3. Tweet MS, Hayes SN, Pitta SR, Simari RD, Lerman A, Lennon RJ, et al. Clinical features, management, and prognosis of spontaneous coronary artery dissection. Circulation 2012;126:579-88.

4. Saw J, Aymong E, Sedlak T, Buller CE, Starovoytov A, Ricci D, et al. Spontaneous coronary artery dissection: Association with predisposing arteriopathies and precipitating stressors and cardiovascular outcomes. Circ Cardiovasc Interv 2014;7:645-55.

5. Aziz S. Spontaneous coronary artery dissection. E J Cardiol Pract 2017;14:1-5

6. Saw J, Mancini GB, Humphries KH. Contemporary review on spontaneous coronary artery dissection. J Am Coll Cardiol 2016;68:297-312.

7. Eleid MF, Guddeti RR, Tweet MS, Lerman A, Singh M, Best PJ, et al. Coronary artery tortuosity in spontaneous coronary artery dissection:
Angiographic characteristics and clinical implications. Circ Cardiovasc Interv 2014;7:656-62.

8. Yeung DF, Saw J. Multiple recurrences of spontaneous coronary artery dissection in a woman with fibromuscular dysplasia. Catheter Cardiovasc Interv 2019;94:702-5.

9. Subramaniam K, Siew SF, Mahmood MS. Sudden cardiac death in a young adult man due to spontaneous coronary artery dissection. Malays J Pathol 2019;41:51-4.

10. Cepas-Guillén PL, Flores-Umanzor EJ, Sabate M, Masotti M. Multivessel spontaneous coronary artery dissection involving the left main coronary artery: A case report. Eur Heart J Case Rep 2019;3:yty168.

11. Phang C, Whitbourn R. Percutaneous coronary intervention in recurrent spontaneous coronary artery dissection: A case report. Eur Heart J Case Rep 2019;3:ytz021.

12. Y-Hassan S, Henareh L. Fibrinolysis-treated myocardial infarction in a patient with missed spontaneous coronary artery dissection associated with takotsubo syndrome: Case report. Eur Heart J Case Rep 2018;2:yty145.

13. Bergen E, Huffer L, Peele M. Survival after spontaneous coronary artery dissection presenting with ventricular fibrillation arrest. J Invasive Cardiol 2005;17:E4-6.

14. Yip A, Saw J. Spontaneous coronary artery dissection-A review. Cardiovasc Diagn Ther 2015;5:37-48.

15. Gilhofer TS, Saw J. Spontaneous coronary artery dissection: A review of complications and management strategies. Expert Rev Cardiovasc Ther 2019;17:275-91.

Funding: None; Conflicts of Interest: None Stated.

How to cite this article: Das D, Acharya D, Singh J, Pramanik S. Spontaneous Spiral Dissection of the Left Anterior Descending Coronary Artery in a Reformed Smoker. Indian J Case Reports. 2021;7(4):155-157. 